\title{
On growth rates of closed permutation classes
}

\author{
Tomáš Kaiser ${ }^{1,3}$ and Martin Klazar²,3 \\ Submitted: Apr 26, 2002; Accepted: Mar 17, 2003; Published: Apr 10, 2003 \\ MR Subject Classifications: 05A16, 05A05
}

\begin{abstract}
A class of permutations $\Pi$ is called closed if $\pi \subset \sigma \in \Pi$ implies $\pi \in \Pi$, where the relation $\subset$ is the natural containment of permutations. Let $\Pi_{n}$ be the set of all permutations of $1,2, \ldots, n$ belonging to $\Pi$. We investigate the counting functions $n \mapsto\left|\Pi_{n}\right|$ of closed classes. Our main result says that if $\left|\Pi_{n}\right|<2^{n-1}$ for at least one $n \geq 1$, then there is a unique $k \geq 1$ such that $F_{n, k} \leq\left|\Pi_{n}\right| \leq F_{n, k} \cdot n^{c}$ holds for all $n \geq 1$ with a constant $c>0$. Here $F_{n, k}$ are the generalized Fibonacci numbers which grow like powers of the largest positive root of $x^{k}-x^{k-1}-\cdots-1$. We characterize also the constant and the polynomial growth of closed permutation classes and give two more results on these.
\end{abstract}

\section{Introduction}

A permutation $\sigma=\left(b_{1}, b_{2}, \ldots, b_{n}\right)$ of $[n]=\{1,2, \ldots, n\}$ contains a permutation $\pi=$ $\left(a_{1}, a_{2}, \ldots, a_{k}\right)$ of $[k]$, in symbols $\sigma \supset \pi$, if $\sigma$ has a (not necessarily consecutive) subsequence of length $k$ whose terms induce the same order pattern as $\pi$. For example, $(3,5,4,2,1,7,8,6,9)$ contains $(2,1,3)$, as $(\ldots, 5, \ldots, 1, \ldots, 6, \ldots)$ or as $(\ldots, 4,2, \ldots, 9)$, but it does not contain $(3,1,2)$.

Let $f(n, \pi)$ be the number of the permutations of $[n]$ not containing $\pi$. The following conjecture was made by R. P. Stanley and H. S. Wilf (it appeared first in print in Bóna $[11,12,13])$.

The Stanley-Wilf conjecture. For every permutation $\pi$, there is a constant $c>0$ such that $f(n, \pi)<c^{n}$ for all $n \geq 1$.

\footnotetext{
${ }^{1}$ Department of Mathematics, University of West Bohemia, Univerzitní 8, 30614 Plzeň, Czech Republic, e-mail: kaisert@kma.zcu.cz. Corresponding author.

${ }^{2}$ Department of Applied Mathematics, Charles University, Malostranské náměstí 25, 11800 Praha 1, Czech Republic, e-mail: klazar@kam.mff.cuni.cz.

${ }^{3}$ Institute for Theoretical Computer Science (ITI), Charles University, Praha, Czech Republic. Supported by project LN00A056 of the Czech Ministry of Education.
} 
It is known to hold for all $\pi$ of length at most 4 (Bóna [13]), for all layered $\pi$ (Bóna [14], see below for the definition of layered permutations), and for all $\pi$ in a weaker form with an almost exponential upper bound (Alon and Friedgut [3]). A permutation $\pi$ of $[n]$ is called layered if $[n]$ can be partitioned into intervals $I_{1}<I_{2}<\ldots<I_{k}$ so that every restriction $\pi \mid I_{i}$ is decreasing and $\pi\left(I_{1}\right)<\pi\left(I_{2}\right)<\ldots<\pi\left(I_{k}\right)$. (We call $\pi$ layered also in the case when $\pi\left(I_{1}\right)>\pi\left(I_{2}\right)>\ldots>\pi\left(I_{k}\right)$ and the restrictions $\pi \mid I_{i}$ are increasing.) Equivalently, $\pi$ is layered (in the former sense) if and only if it contains neither $(2,3,1)$ nor $(3,1,2)$. Other works dealing with the conjecture and/or the containment of permutations are, to name a few, Adin and Roichman [1], Albert et al. [2], Bóna [12], Klazar [20], Stankova-Frenkel and West [30], and West [34].

A class $\Pi$ of permutations is closed if, for every $\pi$ and $\sigma, \pi \subset \sigma \in \Pi$ implies $\pi \in \Pi$. The symbol $\Pi_{n}, n \in \mathbf{N}=\{1,2, \ldots\}$, denotes the set of all permutations in $\Pi$ of length $n$. The counting function of $\Pi$ is the function $n \mapsto\left|\Pi_{n}\right|$ whose value at $n$ is the number of permutations in $\Pi$ of length $n$. For example, $n \mapsto 0$ is the counting function of the empty class $\Pi=\emptyset$, while the (closed) class of all permutations has the counting function $n \mapsto n$ !. The Stanley-Wilf conjecture says, in effect, that except for the latter trivial example, there are no other superexponential counting functions.

A reformulation of the Stanley-Wilf conjecture. Let $\Pi$ be any closed class of permutations different from the class of all permutations. Then $\left|\Pi_{n}\right|<c^{n}$ for all $n \geq 1$ and a constant $c>0$.

Indeed, if $\Pi$ is closed and $\pi \notin \Pi$, then $\left|\Pi_{n}\right| \leq f(n, \pi)$ for all $n \geq 1$. On the other hand, for every $\pi$ the function $n \mapsto f(n, \pi)$ is the counting function of the closed class consisting of all permutations not containing $\pi$.

If one starts to investigate the realm of closed permutation classes from the top, one gets immediately stuck at the question whether every counting function different from the trivial $n \mapsto n$ ! has to be at most exponential. In this article we take the other course and start from the bottom, at the empty class $\Pi=\emptyset$. We shall investigate the counting functions of 'small' closed permutation classes.

We summarize our results and give a few more definitions. Theorem 2.1 points out two simple set-theoretical facts about the set of all closed classes. Theorem 2.2, due to P. Valtr, gives a uniform lower bound on $\liminf _{n \rightarrow \infty} f(n, \pi)^{1 / n}$. Sections 3 and 4 contain our main results. Theorem 3.4 shows that any counting function grows either at most polynomially or at least as the Fibonacci numbers $F_{n}$. Thus $n \mapsto F_{n}$ is the smallest superpolynomial counting function. Theorem 3.8 classifies the possible exponential growth rates below $n \mapsto 2^{n-1}$ : Either $\left|\Pi_{n}\right| \geq 2^{n-1}$ for all $n \geq 1$, or there is a unique $k \geq 1$ such that $\left|\Pi_{n}\right|$ grows, up to a polynomial factor, as the generalized Fibonacci numbers $F_{n, k}$. Theorem 4.2 shows that any counting function is either eventually constant or grows at least as the identity function $n \mapsto n$. Thus $n \mapsto n$ is the smallest unbounded counting function. Theorem 4.4 shows that if the function $n \mapsto\left|\Pi_{n}\right|$ grows polynomially, then it is eventually an integral linear combination of the polynomials $\left(\begin{array}{c}n-i \\ j\end{array}\right)$. The concluding part (Section 5) contains some remarks and comments. 
Recall that $\mathbf{N}$ denotes $\{1,2, \ldots\}$, the set of positive integers, and $[n]$ denotes the set $\{1,2, \ldots, n\}$. More generally, for $a, b \in \mathbf{N}$ and $a \leq b$, the interval $\{a, a+1, \ldots, b\}$ is denoted by $[a, b]$. If $\pi$ is a permutation of $[n]$, we say that $n$ is its length and write $|\pi|=n$. Let $A_{1}, A_{2}, B_{1}, B_{2} \subset \mathbf{N}$ be four finite sets of the same cardinality. We call two bijections $f: A_{1} \rightarrow A_{2}$ and $g: B_{1} \rightarrow B_{2}$ similar iff $f(x)=j(g(h(x)))$ holds for every $x \in A_{1}$, where $h: A_{1} \rightarrow B_{1}$ and $j: B_{2} \rightarrow A_{2}$ are the unique increasing bijections. In other words, using only the order relation we cannot distinguish the graphs of $f$ and $g$. Every bijection between two $n$-element subsets of $\mathbf{N}$ is similar to a unique permutation of $[n]$. For two permutations $\sigma$ and $\pi, \sigma$ contains $\pi$ iff a subset of $\sigma$ (regarded as a set of pairs) is similar to $\pi$. We take the restriction $\pi \mid X$ of a permutation $\pi$ of $[n]$ to a subset $X \subset[n]$ to be the unique permutation similar to the usual restriction. For a set of permutations $X$ we define

$$
\operatorname{Forb}(X)=\{\pi: \pi \text { contains no } \sigma \in X\} .
$$

For any $X$, this is a closed class. Note that for every closed class $\Pi$ there is exactly one set $X$ of permutations pairwise incomparable by $\subset$ (that is, $X$ is an antichain) such that $\Pi=\operatorname{Forb}(X)$; the set $X$ consists of the minimal permutations not in $\Pi$. Thus the closed permutation classes correspond bijectively to antichains of permutations. A function $f: \mathbf{N} \rightarrow \mathbf{N}$ eventually dominates another function $g: \mathbf{N} \rightarrow \mathbf{N}$ iff $f(n) \geq g(n)$ for every $n \geq n_{0}$.

\section{The number of closed classes and a lower bound on $f(n, \pi)$}

If $\Pi$ and $\Pi^{\prime}$ are closed classes of permutations and $\Pi \backslash \Pi^{\prime}$ is finite then, trivially, $n \mapsto\left|\Pi_{n}^{\prime}\right|$ eventually dominates $n \mapsto\left|\Pi_{n}\right|$. By the following theorem, there are uncountably many classes such that this trivial comparison does not apply for any two of them.

Theorem 2.1 (1) There exist $2^{\aleph_{0}}$ (continuum many) distinct closed classes of permutations.

(2) In fact, there exists a set $S$ of $2^{\aleph_{0}}$ closed classes of permutations such that for every $\Pi, \Pi^{\prime} \in S, \Pi \neq \Pi^{\prime}$, both sets $\Pi \backslash \Pi^{\prime}$ and $\Pi^{\prime} \backslash \Pi$ are infinite.

Proof. (1) It is known (see, for example, Spielman and Bóna [29]) that there is an infinite antichain of permutations $A$. Then

$$
\{\operatorname{Forb}(X): X \subset A\}
$$

is a set of $2^{\aleph_{0}}$ closed classes. Indeed, every $\operatorname{Forb}(X)$ is closed and it is easy to see that $X, Y \subset A, X \neq Y$ implies $\operatorname{Forb}(X) \neq \operatorname{Forb}(Y)$.

(2) In fact, if $X, Y \subset A$ and $\pi \in X \backslash Y$ then $\pi \in \operatorname{Forb}(Y) \backslash \operatorname{Forb}(X)$. It suffices to show that there is a system of $2^{\aleph_{0}}$ subsets of $A$ such that the set difference of every two distinct members of the system is infinite. For the notational convenience we identify $A$ with $\mathbf{N}$. 
Recall that for $X \subset \mathbf{N}=A$, the upper and lower asymptotic densities of $X$ are defined as

$$
\bar{d}(X)=\limsup _{n \rightarrow \infty} \frac{|X \cap[n]|}{n} \text { and } \underline{d}(X)=\liminf _{n \rightarrow \infty} \frac{|X \cap[n]|}{n} .
$$

For every real constant $c, 0<c<\frac{1}{2}$, we select a subset $X_{c} \subset \mathbf{N}=A$ such that $\bar{d}\left(X_{c}\right)=$ $1-c$ and $\underline{d}\left(X_{c}\right)=c$. Then

$$
S=\left\{\operatorname{Forb}\left(X_{c}\right): 0<c<\frac{1}{2}\right\}
$$

is a set of $2^{\aleph_{0}}$ closed classes with the stated property. Indeed, for every two real constants $c, d \in\left(0, \frac{1}{2}\right), c \neq d$, the set $X_{c} \backslash X_{d}$ is infinite because for $X, Y \subset \mathbf{N}$ with $X \backslash Y$ finite one has $\underline{d}(X) \leq \underline{d}(Y)$ and $\bar{d}(X) \leq \bar{d}(Y)$.

Of course, there is nothing special about permutations in the previous theorem. It holds for the closed classes in any countably infinite poset that has an infinite antichain. Do there exist two closed classes of permutations such that their counting functions are incomparable by the eventual dominance? Are there $2^{\aleph_{0}}$ such closed permutation classes?

We take the opportunity to include an unpublished lower bound on the size of a class characterized by a forbidden permutation. The following theorem and its proof are due to Pavel Valtr [33] and are reproduced here with his kind permission.

Theorem 2.2 Let $c$ be any constant such that $0<c<\mathrm{e}^{-3}=0.04978 \ldots$ Then for any permutation $\pi$ of length $k$, where $k>k_{0}=k_{0}(c)$, we have

$$
\liminf _{n \rightarrow \infty} f(n, \pi)^{1 / n}>c k^{2}
$$

Proof. Let $\pi$ be a permutation of length $k$. A random permutation $\tau$ of length $m$ contains $\pi$ with probability

$$
\operatorname{Pr}[\tau \supset \pi] \leq \frac{1}{k !}\left(\begin{array}{c}
m \\
k
\end{array}\right)<\frac{m^{k}}{(k !)^{2}} .
$$

We set $m=\left\lfloor d k^{2}\right\rfloor$ where $0<d<\mathrm{e}^{-2}$ is a constant. Then, by the Stirling asymptotics, this probability goes to 0 with $k \rightarrow \infty$ and for all sufficiently large $k$ we have

$$
f(m, \pi)>\frac{m !}{2} .
$$

We can assume that $\pi$ cannot be split as $[k]=I \cup J, I<J$, where both intervals $I$ and $J$ are nonempty and $\pi(I)<\pi(J)$. (Otherwise we replace $\pi$ with the reversed permutation.) Let $n \in \mathbf{N}$ and $n=m t+u$, where $t \geq 0$ and $0 \leq u<m$ are integers. It follows that none of the $f(m, \pi)^{t} f(u, \pi)$ permutations

$$
\left(b_{1}, \ldots, b_{u}, d_{1}+a_{1}^{1}, \ldots, d_{1}+a_{m}^{1}, d_{2}+a_{1}^{2}, \ldots, d_{2}+a_{m}^{2}, \ldots, d_{t}+a_{1}^{t}, \ldots, d_{t}+a_{m}^{t}\right)
$$

of length $n$, where $d_{i}=u+(i-1) m$ and $\left(b_{1}, \ldots, b_{u}\right)$ and $\left(a_{1}^{i}, \ldots, a_{m}^{i}\right)$ are permutations not containing $\pi$, contains $\pi$. Since $m !>(m / e)^{m}$ for large $k$,

$$
f(n, \pi)^{1 / n} \geq f(m, \pi)^{t / n}>\left(\frac{m !}{2}\right)^{t / n}>\left(\frac{m !}{2}\right)^{1 / m-1 / n}>\frac{2^{1 / n-1 / m}}{(m !)^{1 / n}} \cdot \frac{m}{\mathrm{e}} .
$$


By the choice of $m$, for any $\varepsilon>0$ and $k>k_{0}=k_{0}(\varepsilon)$,

$$
\liminf _{n \rightarrow \infty} f(n, \pi)^{1 / n}>\frac{(1-\varepsilon) d}{\mathrm{e}} \cdot k^{2} .
$$

Arratia [4] proved that $\lim _{n \rightarrow \infty} f(n, \pi)^{1 / n}$ always exists, and therefore in the previous bound we can replace lim inf with lim. For a general permutation $\pi$ of length $k$ the bound is best possible, up to the constant $c$, because

$$
f(n,(1,2, \ldots, k)) \leq \frac{1}{(k-1) !} \sum_{\substack{0 \leq i_{1}, \ldots, i_{k-1} \\
i_{1}+\cdots+i_{k-1}=n}}\left(\begin{array}{c}
n \\
i_{1}, \ldots, i_{k-1}
\end{array}\right)^{2} \leq \frac{(k-1)^{2 n}}{(k-1) !}
$$

The first inequality follows from the fact that by Dilworth's theorem, every permutation with no increasing subsequence of length $k$ can be partitioned into at most $k-1$ decreasing subsequences. The second inequality follows by the multinomial theorem. Thus $\lim _{n \rightarrow \infty} f(n,(1,2, \ldots, k))^{1 / n} \leq(k-1)^{2}$. By the exact asymptotics found by Regev [27], $\lim _{n \rightarrow \infty} f(n,(1,2, \ldots, k))^{1 / n}=(k-1)^{2}$.

Theorem 2.2 improves a result of M. Petkovšek, see Wilf [35, Theorem 4], that gives a linear lower bound on $\liminf _{n \rightarrow \infty} f(n, \pi)^{1 / n}$, namely

$$
\liminf _{n \rightarrow \infty} f(n, \pi)^{1 / n} \geq k-1
$$

where $k$ is the length of $\pi$.

\section{Below $n \mapsto 2^{n-1}$ - the Fibonacci growths}

In this section we prove Theorem 3.8 which characterizes the exponential growth rates possible for the closed permutation classes $\Pi$ satisfying $\left|\Pi_{n}\right|<2^{n-1}$ for at least one $n \geq 1$. For the proof of the following classic result see, for example, Lovász [24, Problem 14.25].

Theorem 3.1 (Erdős-Szekeres) Every sequence of $n$ integers has a monotone subsequence of length at least $n^{1 / 2}$.

A permutation $\pi,|\pi|=n$, has $k$ alternations if there are $2 k$ indices $1 \leq i_{1}<j_{1}<i_{2}<$ $j_{2}<\ldots<i_{k}<j_{k} \leq n$ such that

$$
\pi\left(\left\{i_{1}, i_{2}, \ldots, i_{k}\right\}\right)>\pi\left(\left\{j_{1}, j_{2}, \ldots, j_{k}\right\}\right)
$$

A closed permutation class $\Pi$ unboundedly alternates if for every $k \geq 1$ there is a $\pi \in \Pi$ such that $\pi$ or $\pi^{-1}$ has $k$ alternations.

Lemma 3.2 If a closed permutation class $\Pi$ unboundedly alternates, then $\left|\Pi_{n}\right| \geq 2^{n-1}$ for every $n \geq 1$. 
Proof. We suppose that for any $k$ there is a $\pi \in \Pi$ with $k$ alternations; the case with $\pi^{-1}$ is analogous. Using Theorem 3.1 and the fact that $\Pi$ is closed, we see that for every $n \geq 1$ there is a $\pi \in \Pi_{2 n+1}$ such that the restriction $\pi \mid\{2 i-1: 1 \leq i \leq n+1\}$ is monotone, and $\pi(i)>\pi(j)$ whenever $i$ is odd and $j$ is even. We may assume that the restriction is increasing; the other case when it is decreasing is quite similar. Since $\Pi$ is closed, there is for every $X \subset[2, n]$ some $\pi_{X} \in \Pi_{n}$ such that $\pi_{X}(i)>\pi_{X}(1) \Leftrightarrow i \in X$. Distinct $X$ give distinct permutations $\pi_{X}$ and thus $\left|\Pi_{n}\right| \geq 2^{n-1}$.

A word $u=u_{1} u_{2} \ldots u_{n}$ has no immediate repetitions if $u_{i} \neq u_{i+1}$ for each $1 \leq i \leq n-1$. We say that $u$ is alternating if $u=a b a b a \ldots$ for two distinct symbols $a$ and $b$. For a word $u$ we denote $\ell(u)$ the maximum length of an alternating subsequence of $u$. Let

$$
W_{m, l, n}=\left\{u \in[m]^{*}:|u|=n \& \ell(u) \leq l\right\}
$$

be the set of all words over the alphabet $[m]$ of length $n$ which have no alternating subsequence of length $l+1$. Claim (1) of the following lemma is a result of Davenport and Schinzel [15].

Lemma 3.3 (1) If $u=u_{1} u_{2} \ldots u_{n}$ is a word over $[m]$ which has no immediate repetitions and satisfies $\ell(u) \leq l$, then

$$
n \leq\left(\begin{array}{c}
m \\
2
\end{array}\right)(l-1)+1 .
$$

(2) For every $m, l, n \geq 1$ we have

$$
\left|W_{m, l, n}\right| \leq(m+1)^{c} \cdot n^{c}
$$

where $c=\left(\begin{array}{c}m \\ 2\end{array}\right)(l-1)+1$.

(3) Suppose that the alphabet $[m]$ is partitioned into $r$ subalphabets $A_{1}, \ldots, A_{r}$ and $u$ is a word over $[\mathrm{m}]$ such that every subword $v_{i}$ of $u$ consisting of the occurrences of the letters in $A_{i}$ satisfies $\ell\left(v_{i}\right) \leq l$. Then $u$ can be split into $t$ intervals $u=I_{1} I_{2} \ldots I_{t}$ such that every $I_{i}$ uses at most one letter from every $A_{j}$, and

$$
t \leq 2\left(\begin{array}{c}
m \\
2
\end{array}\right)(l-1)+2 .
$$

Proof. (1) Let $u=u_{1} u_{2} \ldots u_{n}$ be over $[m]$, without immediate repetitions, and let $n \geq\left(\begin{array}{c}m \\ 2\end{array}\right)(l-1)+2$. By the pigeon-hole principle, some $l$ of the $n-1$ two-element sets $\left\{u_{i}, u_{i+1}\right\}$ must coincide. It is easy to see that the corresponding positions in $u$ contain an $l+1$-element alternating subsequence.

(2) Every word $u \in W_{m, l, n}$ splits uniquely into intervals $u=I_{1} I_{2} \ldots I_{t}$ such that $I_{i}=a_{i} a_{i} \ldots a_{i}$ consists of repetitions of a single letter $a_{i}$ and $a_{i} \neq a_{i+1}$ for $1 \leq i \leq t-1$. Contracting every $I_{i}$ into one term, we obtain a word $u^{*}$ over $[m],\left|u^{*}\right|=t$, with $\ell\left(u^{*}\right) \leq l$ 
and no immediate repetitions. By $(1), t \leq\left(\begin{array}{c}m \\ 2\end{array}\right)(l-1)+1=c$. Clearly, $u^{*}$ and the composition $\left|I_{1}\right|,\left|I_{2}\right|, \ldots,\left|I_{t}\right|$ of $n$ determine $u$ uniquely. Thus

$$
\left|W_{m, l, n}\right| \leq\left(\# u^{*}\right) \cdot n^{c} \leq(m+1)^{c} \cdot n^{c} .
$$

(3) We consider the unique splitting $u=I_{1} I_{2} \ldots I_{t}$, where $I_{1}$ is the longest initial interval of $u$ using at most one letter from every $A_{j}, I_{2}$ is the longest following interval with the same property, etc. Note that every pair $I_{i} I_{i+1}$ has a subsequence $a, b$ (where $b$ is the first term of $I_{i+1}$ ) such that $a, b \in A_{j}$ for some $j$ and $a \neq b$. Now arguing similarly as in (1), we see that

$$
t \leq 2\left(\begin{array}{c}
m \\
2
\end{array}\right)(l-1)+2
$$

The shifted Fibonacci numbers $\left(F_{n}\right)_{n \geq 1}=(1,2,3,5,8,13,21, \ldots)$ are defined by $F_{1}=$ $1, F_{2}=2$, and $F_{n}=F_{n-1}+F_{n-2}$ for $n \geq 3$. The explicit formula is

$$
F_{n}=\frac{1}{\sqrt{5}}\left(\left(\frac{1+\sqrt{5}}{2}\right)^{n+1}-\left(\frac{1-\sqrt{5}}{2}\right)^{n+1}\right)
$$

By induction, $F_{n} \leq 2^{n-1}$ for every $n \geq 1$.

The next theorem identifies the jump from the polynomial to the exponential growth and shows that $n \mapsto F_{n}$ is the first superpolynomial growth rate. Although it is fully subsumed in the more general Theorem 3.8, we give a sketch of the proof. We think that it may be interesting and instructive for the reader to compare how the concepts used here develop later in the more complicated proof of Theorem 3.8.

Theorem 3.4 Let $\Pi$ be any closed class of permutations. Then exactly one of the following possibilities holds.

(1) There is a constant $c>0$ such that $\left|\Pi_{n}\right| \leq n^{c}$ for all $n \geq 1$.

(2) $\left|\Pi_{n}\right| \geq F_{n}$ for all $n \geq 1$.

Proof. (Extended sketch.) We split any permutation $\pi$ into $\pi=S_{1} S_{2} \ldots S_{m}$ where $S_{1}$ is the longest initial monotone segment, $S_{2}$ is the longest following monotone segment, and so on. We mark the elements in $S_{i}$ by $i$ and read the marks from bottom to top (that is, from left to right in $\left.\pi^{-1}\right)$. In this way, we obtain a word $u(\pi)$ over the alphabet $[m]$, where $m=m(\pi)$ is the number of the monotone segments $S_{i}$. For example,

$$
\text { if } \pi=(3,5,4,2,1,7,8,6,9) \text { then } m(\pi)=4 \text { and } u(\pi)=221214334
$$

because $S_{1}=3,5, S_{2}=4,2,1, S_{3}=7,8$, and $S_{4}=6,9$. Note that $\pi$ is determined uniquely by $u(\pi)$ and an $m$-tuple of signs $( \pm, \pm, \ldots, \pm)$ in which + indicates an increasing segment and - a decreasing one. For every pair $S_{i}, S_{i+1}$ we fix an interval $T_{i}=T_{\pi, i}=$ 
$[\min \{a, b, c\}, \max \{a, b, c\}]$ where $a, b, c$ is a non-monotone subsequence of $S_{i} S_{i+1}$ (such a subsequence certainly exists). In our example, for $i=3$ we may set $a, b, c=7,8,6$ and $T_{3}=[6,8]$.

For a closed permutation class $\Pi$ and $\pi$ ranging over $\Pi$ we distinguish four cases. Case 1a: $m(\pi)$ is bounded and so is $\ell(u(\pi))$. Case 1b: $m(\pi)$ is bounded and $\ell(u(\pi))$ is unbounded. Case 2a: $m(\pi)$ is unbounded and the maximum number of mutually intersecting intervals in the system $S(\pi)=\left\{T_{\pi, 1}, T_{\pi, 3}, T_{\pi, 5}, \ldots\right\}$ is unbounded as well. Case 2b: $m(\pi)$ is unbounded and so is the maximum number of mutually disjoint intervals in the system $S(\pi)$.

In case 1a we use (2) of Lemma 3.3 and deduce the polynomial upper bound of claim (1). In case $1 b$, the class $\Pi$ unboundedly alternates and, by Lemma $3.2,\left|\Pi_{n}\right| \geq 2^{n-1} \geq F_{n}$. In case $2 \mathrm{a}$, the class $\Pi$ again unboundedly alternates and $\left|\Pi_{n}\right| \geq 2^{n-1} \geq F_{n}$. In case $2 \mathrm{~b}$, it follows by Theorem 3.1 and the definition of $T_{\pi, i}$, that either for every $n \geq 1$ we have $(2,1,4,3,6,5, \ldots, 2 n, 2 n-1) \in \Pi$ or for every $n \geq 1$ we have $(2 n-1,2 n, 2 n-3,2 n-$ $2, \ldots, 1,2) \in \Pi$. Using the fact that $\Pi$ is closed, we conclude that in this case, $\left|\Pi_{n}\right| \geq F_{n}$.

To state Theorem 3.8, we need a few more definitions and lemmas. For $k$ an integer and $F$ a power series, $\left[x^{k}\right] F$ denotes the coefficient at $x^{k}$ in $F$. We define the family of generalized Fibonacci numbers $F_{n, k} \in \mathbf{N}$, where $k \geq 1$ and $n$ are integers, by

$$
F_{n, k}=\left[x^{n}\right] \frac{1}{1-x^{k}-x^{k-1}-\cdots-x} .
$$

In particular, $F_{n, 1}=1$ for every $n \geq 1$ and $F_{n, 2}=F_{n}$. More generally, $F_{n, k}=0$ for $n<0$, $F_{0, k}=1$, and

$$
F_{n, k}=F_{n-1, k}+F_{n-2, k}+\cdots+F_{n-k, k} \text { for } n>0 .
$$

Lemma 3.5 Let $k \geq 1$ be fixed.

(1) For $n \rightarrow \infty$, we have the asymptotics

$$
F_{n, k}=c_{k} \alpha_{k}^{n}+O\left(\beta_{k}^{n}\right), c_{k}=\frac{\alpha_{k}^{k}\left(\alpha_{k}-1\right)}{\alpha_{k}^{k+1}-k},
$$

where $\alpha_{k}$ is the largest positive real root of $x^{k}-x^{k-1}-x^{k-2}-\cdots-1$ and $\beta_{k}$ is a constant such that $0<\beta_{k}<\alpha_{k}$.

(2) The roots $\alpha_{k}$ satisfy inequalities $1=\alpha_{1}<\alpha_{2}<\alpha_{3}<\ldots<2$, and $\alpha_{k} \rightarrow 2$ as $k \rightarrow \infty$.

(3) For all integers $m$ and $n$,

$$
F_{m, k} \cdot F_{n, k} \leq F_{m+n, k}
$$

(4) For every $n \geq 1$ we have

$$
F_{n, k} \leq 2^{n-1} \quad \text { and } \quad F_{n, n}=2^{n-1}
$$


Proof. (1) Since

$$
\sum_{n \geq 0} F_{n, k} x^{n}=\frac{1}{1-x^{k}-x^{k-1}-\cdots-x},
$$

the asymptotics of $F_{n, k}$ follows by the standard technique of decomposing rational functions into partial fractions (see, for example, Stanley [31, p. 202]). We need to prove only that $\alpha_{k}$ is a simple root of the reciprocal polynomial $p_{k}(x)=x^{k}-x^{k-1}-x^{k-2}-\cdots-1$ and that on the complex circle $|z|=\alpha_{k}$, the polynomial $p_{k}$ has no other root besides $\alpha_{k}$. The constant $\beta_{k}$ can then be set to $\varepsilon+$ the second largest modulus of a root of $p_{k}$. The form of the coefficient $c_{k}$ follows by a simple manipulation from the identity $c_{k}=\alpha_{k}^{k-1} / p_{k}^{\prime}\left(\alpha_{k}\right)$ provided by the partial fractions decomposition.

Clearly, $1 \leq \alpha_{k}<2$. Since $x p_{k}^{\prime}-k p_{k}=x^{k-1}+2 x^{k-2}+\cdots+k$, we have $p_{k}^{\prime}\left(\alpha_{k}\right)>$ $k(k+1) / 4$ and $\alpha_{k}$ is a simple root of $p_{k}$. Since $p_{k}=\left(x^{k+1}-2 x^{k}+1\right) /(x-1), p_{k}(x)=0$ is equivalent to $x^{k}=1 /(2-x)$. It is clear that no $z,|z|=\alpha_{k}, z \neq \alpha_{k}$, satisfies this equation.

(2) This is immediate from the identity $\alpha_{k}^{k}=1 /\left(2-\alpha_{k}\right)$ used in (1).

(3) and (4): These are easy to verify inductively by the recurrence for $F_{n, k}$. We only prove (3). We proceed by induction on $m+n$. For $m<0$ or $n<0$ the inequality is true. It also holds for $m=n=0$. Let $m \geq 0$ and $n \geq 1$. Then

$$
F_{m, k} F_{n, k}=F_{m, k} \sum_{i=n-k}^{n-1} F_{i, k} \leq \sum_{i=m+n-k}^{m+n-1} F_{i, k}=F_{m+n, k} .
$$

We list approximate values of the first few roots $\alpha_{k}$ :

\begin{tabular}{l||l|l|l|l|l|l}
$k$ & 2 & 3 & 4 & 5 & 6 & 10 \\
\hline$\alpha_{k}$ & 1.61803 & 1.83928 & 1.92756 & 1.96594 & 1.98358 & 1.99901
\end{tabular}

Let $A$ be a finite alphabet equipped with a weight function $w: A \rightarrow \mathbf{N}$. The weight $w(u)$ of a word $u=u_{1} u_{2} \ldots u_{m} \in A^{*}$ is the sum $w\left(u_{1}\right)+w\left(u_{2}\right)+\cdots+w\left(u_{m}\right)$. We set

$$
p(w, n)=\#\left\{u \in A^{*}: w(u)=n\right\} .
$$

Lemma 3.6 Let $k \geq 1$ be fixed.

(1) If $A=\left\{a_{1}, a_{2}, \ldots, a_{k}\right\}$ and $w\left(a_{i}\right)=i$ for $i=1, \ldots, k$, then $p(w, n)=F_{n, k}$ for every $n \geq 1$.

(2) If $A=\left\{a_{1}, a_{2}, \ldots, a_{k+1}\right\}$ and $w\left(a_{i}\right)=i$ for $i=1, \ldots, k$ and $w\left(a_{k+1}\right)=k$, then $p(w, n) \geq 2^{n-1}$ for every $n \geq 1$.

Proof. In the general situation we have the identity

$$
\sum_{n=0}^{\infty} p(w, n) x^{n}=\frac{1}{1-\sum_{a \in A} x^{w(a)}} .
$$


Now (1) is clear since then $\sum_{a \in A} x^{w(a)}=x^{k}+x^{k-1}+\cdots+x$.

In (2), we have

$$
\sum_{n=0}^{\infty} p(w, n) x^{n}=\frac{1}{1-\left(2 x^{k}+x^{k-1}+\cdots+x\right)}
$$

and the inequality $p(w, n) \geq 2^{n-1}$ follows by induction from the recurrence

$$
p(w, n)=p(w, n-1)+\cdots+p(w, n-k+1)+2 p(w, n-k)(n>0)
$$

starting from $p(w, n)=0$ for $n<0$ and $p(w, 0)=1$.

In (2), one might be interested in a more precise bound. Since $1-\left(2 x^{k}+x^{k-1}+\cdots+x\right)=$ $(1-2 x)\left(x^{k-1}+x^{k-2}+\cdots+1\right)$, the decomposition into partial fractions gives

$$
p(w, n)=\left[x^{n}\right]\left(\frac{\alpha}{1-2 x}+\frac{\beta_{1}}{1-x / \zeta_{1}}+\cdots+\frac{\beta_{k-1}}{1-x / \zeta_{k-1}}\right)
$$

where $\alpha, \beta_{1}, \ldots \beta_{k-1} \in \mathbf{C}$ are suitable constants and $\zeta_{i}$ are the $k$-th roots of unity distinct from 1. Thus $\alpha=1 / \sum_{i=0}^{k-1}\left(\frac{1}{2}\right)^{i}$ and, for $n \rightarrow \infty$, we obtain the asymptotics

$$
p(w, n)=\left(\frac{1}{2}+\frac{1}{2^{k+1}-2}\right) \cdot 2^{n}+O(1) .
$$

An upward splitting of a permutation $\pi,|\pi|=n$, is a partition $[n]=[1, r] \cup[r+1, n]$, where $1 \leq r<n$, such that $\pi([1, r])<\pi([r+1, n])$. If $\pi$ has no upward splitting, we say that $\pi$ is upward indecomposable. The set $\mathrm{Ind}^{+}$consists of all upward indecomposable permutations and $\operatorname{Ind}_{n}^{+}=\left\{\pi \in \operatorname{Ind}^{+}:|\pi|=n\right\}$. Every permutation $\pi$ of $[n]$ has a unique decomposition $\pi\left|I_{1}, \ldots, \pi\right| I_{m}$, called the upward decomposition of $\pi$, in which $I_{1}<I_{2}<\ldots<I_{m}$ are intervals partitioning $[n]$ such that $\pi\left(I_{1}\right)<\pi\left(I_{2}\right)<\ldots<\pi\left(I_{m}\right)$ and every restriction $\pi \mid I_{i}$ is upward indecomposable. (This decomposition can be obtained by iterating the upward splittings.) We call the permutations $\pi \mid I_{i}$ the upward blocks of $\pi$. Notions symmetric to these are obtained in the obvious way, replacing the appropriate signs $<$ by the opposite signs $>$. Thus we get the definitions of downward splittings, downward indecomposability, downward decompositions, downward blocks, and the sets Ind $^{-}$and $\operatorname{Ind}_{n}^{-}$.

We prove that one can delete an entry from any upward indecomposable permutation in such a way that the result is upward indecomposable. Needless to say, the same holds for downward indecomposable permutations.

Lemma 3.7 For every $\pi \in \operatorname{Ind}_{n}^{+}, n>1$, there is some $i \in[n]$ such that $\pi \mid([n] \backslash\{i\})$ is in $\operatorname{Ind}_{n-1}^{+}$.

Proof. For a permutation $\pi$ of $[n]$ and $i \in[n]$ we say that $i$ is a record of $\pi$ if $\pi(j)<\pi(i)$ for every $j<i$. Let $1=r_{1}<r_{2}<\ldots<r_{m} \leq n$ be the records of $\pi$. It is easy to see that $\pi$ is upward indecomposable if and only if for every $i=1,2, \ldots, m-1$ there is a $j$, 
$r_{i+1}<j \leq n$, with $\pi(j)<\pi\left(r_{i}\right)$. Suppose that $\pi,|\pi|=n \geq 2$, is upward indecomposable and consider the set $A=\left\{i \in[n]: r_{m}<i \leq n \& \pi(i)>\pi\left(r_{m-1}\right)\right\}$; if $m=1$, we set $A=[2, n]$. If $A \neq \emptyset$, the deletion of any $i \in A$ leaves an upward indecomposable permutation. If $A=\emptyset$, we delete $i=r_{m}$.

We remark that it is easy to find examples of permutations of arbitrary length such that the statement of Lemma 3.7 is satisfied with only two indices $i$.

If $\pi$ is a permutation, $|\pi|=n$, and $I_{1}<I_{2}<\ldots<I_{m}$ is a partition of $[n]$ into $m$ nonempty intervals, we associate with $\pi$ (as in the sketched proof of Theorem 3.4) the word $u(\pi)=u_{1}, u_{2}, \ldots, u_{n}$ over the alphabet $[m]$ by setting $u_{i}=j$ if $\pi^{-1}(i) \in I_{j}$. Note that $\pi$ is uniquely determined by $u(\pi)$ and the $m$ restrictions $\pi \mid I_{i}$.

Also, we associate with $\pi$ the word $v^{+}(\pi)$ over the alphabet $\mathrm{Ind}^{+}$describing the upward decomposition of $\pi$. By $h^{+}(\pi) \in \mathbf{N}$ we denote the maximum size of an upward block of $\pi$ appearing in the upward decomposition of $\pi$. Thus if $h^{+}(\pi)=k$ then $v^{+}(\pi) \in$ $\left(\operatorname{Ind}_{1}^{+} \cup \ldots \cup \operatorname{Ind}_{k}^{+}\right)^{*}$. In the analogous way we define $v^{-}(\pi)$ and $h^{-}(\pi)$.

Theorem 3.8 Let $\Pi$ be any closed class of permutations. Then either $\Pi$ is finite, or exactly one of the following possibilities holds.

(1) There is a unique $k \geq 1$ and a constant $c>0$ such that $F_{n, k} \leq\left|\Pi_{n}\right| \leq F_{n, k} \cdot n^{c}$ for all $n \geq 1$.

(2) $\left|\Pi_{n}\right| \geq 2^{n-1}$ for all $n \geq 1$.

Proof. The $k$-decomposition, where $k \geq 2$ is an integer, of a permutation $\pi,|\pi|=n$, is the unique partition of $[n]$ into the intervals $U_{1}<U_{2}<\ldots<U_{m}$ such that $U_{1}$ is the longest initial interval of $[n]$ with $h^{+}\left(\pi \mid U_{1}\right)<k$ or $h^{-}\left(\pi \mid U_{1}\right)<k, U_{2}$ is the longest following interval with the same property, etc. We call the intervals $U_{i}$ the $k$-segments of $\pi$. The number $m$ of $k$-segments of $\pi$ is denoted by $s_{k}(\pi)$.

Let $\Pi$ be an infinite closed permutation class. Let $s_{k}(\Pi)=\max \left\{s_{k}(\pi): \pi \in \Pi\right\}$. We set $s_{1}(\Pi)=\infty$. For every fixed $k \geq 1$ we prove the following claims.

Claim A. If $s_{k}(\Pi)=\infty$ then $\left|\Pi_{n}\right| \geq F_{n, k}$ for every $n \geq 1$.

Claim B. If $s_{k}(\Pi)<\infty$ then either $\left|\Pi_{n}\right| \geq 2^{n-1}$ for every $n \geq 1$ or $\left|\Pi_{n}\right| \leq F_{n, k-1} \cdot c_{1} n^{c_{2}}$ for every $n \geq 1$ and some constants $c_{1}, c_{2}>0$.

This will prove the theorem. To see this, note that either $s_{k}(\Pi)=\infty$ for every $k \geq 1$ or there is a $k \geq 1$ such that $s_{k}(\Pi)=\infty$ but $s_{k+1}(\Pi)<\infty$. In the former case, claim A implies that $\left|\Pi_{n}\right| \geq F_{n, n}=2^{n-1}$ for every $n \geq 1$ (by (4) of Lemma 3.5). In the latter case, we apply claim A with $k$ and claim $\mathrm{B}$ with $k+1$ and conclude that either again $\left|\Pi_{n}\right| \geq 2^{n-1}$ for every $n \geq 1$ or that $F_{n, k} \leq\left|\Pi_{n}\right| \leq F_{n, k} \cdot n^{c}$ for every $n \geq 1$ ( $c_{1}$ was absorbed in the enlarged $c_{2}$ ).

Proof of Claim A. For a $\pi \in \Pi$ with the $k$-segments $U_{1}<U_{2}<\ldots<U_{s_{k}(\pi)}$, we set $T_{\pi, i}=\left[\min \left(\pi\left(U_{i} U_{i+1}\right)\right), \max \left(\pi\left(U_{i} U_{i+1}\right)\right)\right]$. Note that, by the definition of $k$-segments and 
Lemma 3.7, every restriction $\pi \mid U_{i} U_{i+1}$ contains a member of $\operatorname{Ind}_{k}^{+}$and a member of $\operatorname{Ind}_{k}^{-}$. We consider the system of intervals

$$
S(\pi)=\left\{T_{\pi, 1}, T_{\pi, 3}, T_{\pi, 5}, \ldots, T_{\pi, r}\right\}, \quad \text { where } r=2\left\lceil\left(s_{k}(\pi)-1\right) / 2\right\rceil-1 .
$$

By the Ramsey theorem, either for every $m \geq 1$ there is a $\pi \in \Pi$ such that $S(\pi)$ contains $m$ mutually intersecting intervals or for every $m \geq 1$ the same holds with mutually disjoint intervals. In the former case, it is easy to see that $\pi$ must have at least $m / 2$ alternations, since all members of a system of mutually intersecting intervals must have a point in common. By Lemma 3.2 and (4) of Lemma 3.5, $\left|\Pi_{n}\right| \geq 2^{n-1} \geq F_{n, k}$ for every $n \geq 1$.

In the latter case, for every $m \geq 1$ there is a $\pi \in \Pi$ for which $[|\pi|]$ can be partitioned into $m$ intervals $I_{1}<I_{2}<\ldots<I_{m}$ such that every restriction $\pi \mid I_{i}$ contains a member of $\operatorname{Ind}_{k}^{+}$and a member of $\operatorname{Ind}_{k}^{-}$, and for every $i \neq j$ we have $\pi\left(I_{i}\right)>\pi\left(I_{j}\right)$ or $\pi\left(I_{i}\right)<\pi\left(I_{j}\right)$. By Theorem 3.1, we may assume that $\pi\left(I_{1}\right)<\pi\left(I_{2}\right)<\ldots<\pi\left(I_{m}\right)$ or $\pi\left(I_{1}\right)>\pi\left(I_{2}\right)>$ $\ldots>\pi\left(I_{m}\right)$. Let $\pi\left(I_{1}\right)<\pi\left(I_{2}\right)<\ldots<\pi\left(I_{m}\right)$; the other case is similar. Since $m$ may be arbitrarily large and $\left|\operatorname{Ind}_{k}^{+}\right| \leq k$ !, we may use the pigeon-hole principle and assume that there is one fixed $\sigma \in \operatorname{Ind}_{k}^{+}$that is contained, for every $m \geq 1$, in every $\pi \mid I_{i}, 1 \leq i \leq m$. By Lemma 3.7, there is a set of permutations $\Sigma=\left\{\sigma_{1}, \sigma_{2}, \ldots, \sigma_{k}\right\}$ such that $\sigma_{i} \in \operatorname{Ind}_{i}^{+}$, $\sigma_{i} \subset \sigma_{i+1}$, and $\sigma_{k}=\sigma$. Since $\Pi$ is closed, for every word $u$ over the alphabet $\Sigma$ there is a $\tau \in \Pi$ (contained in $\pi$ ) such that $v^{+}(\tau)=u$. Clearly, different words $u$ determine different permutations $\tau$. Using (1) of Lemma 3.6 (where the weight function is $w\left(\sigma_{i}\right)=\left|\sigma_{i}\right|=i$ ), we conclude that $\left|\Pi_{n}\right| \geq F_{n, k}$ for every $n \geq 1$. This finishes the proof of Claim A.

Proof of Claim B. We have $k \geq 2$ and there is a constant $K$ such that $s_{k}(\pi) \leq K$ for every $\pi \in \Pi$. If $\pi \in \Pi_{n}$ and $U_{1}<U_{2}<\ldots<U_{s_{k}(\pi)}$ is the partition of $[n]$ into the $k$-segments of $\pi$, we consider the word $u(\pi)$ over $[K]$ as defined above the theorem.

For $1 \leq i \leq s_{k}(\pi)$ and $1 \leq j \leq k-1$ we define $v_{i, j}^{+}(\pi)$ as the subword of $v^{+}\left(\pi \mid U_{i}\right)$ consisting of the occurrences of the letters from the subalphabet $\operatorname{Ind}_{j}^{+}$. The word $v_{i, j}^{-}(\pi)$ is defined in the obvious symmetric manner. Recall that $\ell(u)$ is the length of the longest alternating subsequence of $u$. Let $\ell(u(\Pi))=\max \{\ell(u(\pi)): \pi \in \Pi\}$ and, for $1 \leq i \leq K$ and $1 \leq j \leq k-1, \ell\left(v_{i, j}^{+}(\Pi)\right)=\max \left\{\ell\left(v_{i, j}^{+}(\pi)\right): \pi \in \Pi\right\}$. The quantity $\ell\left(v_{i, j}^{-}(\Pi)\right)$ is defined analogously. (For $i>s_{k}(\pi)$ we set $\ell\left(v_{i, j}^{+}(\pi)\right)=\ell\left(v_{i, j}^{-}(\pi)\right)=0$.) We distinguish two complementary cases.

Case B1. One of the $2 K(k-1)+1$ quantities $\ell(u(\Pi)), \ell\left(v_{i, j}^{+}(\Pi)\right)$, and $\ell\left(v_{i, j}^{-}(\Pi)\right)$ equals $\infty$.

We prove that then always

$$
\left|\Pi_{n}\right| \geq 2^{n-1} \text { for all } n \geq 1
$$

For unbounded $\ell(u(\pi))$ we can find a $\pi \in \Pi$ with as many alternations in $\pi^{-1}$ as we wish and thus $\left|\Pi_{n}\right| \geq 2^{n-1}$ for every $n \geq 1$ by Lemma 3.2. For unbounded $\ell\left(v_{i, j}^{+}(\pi)\right)$ (the argument for $v_{i, j}^{-}(\pi)$ is the same) there is a $j \in[k-1]$ (in fact, necessarily $j \in[3, k-1]$ ) and two distinct permutations $\tau, \sigma \in \operatorname{Ind}_{j}^{+}$such that for every alternating word $v$ over 
$\{\sigma, \tau\}$ there is a $\pi \in \Pi$ with $v^{+}(\pi)=v$. Using Lemma 3.7 again, we can take a set of permutations $\Sigma=\left\{\sigma_{1}, \sigma_{2}, \ldots, \sigma_{j}\right\}$ such that $\sigma_{i} \in \operatorname{Ind}_{i}^{+}, \sigma_{i} \subset \sigma_{i+1}$, and $\sigma_{j}=\sigma$. Since $\Pi$ is closed, for every word $v$ over the alphabet $\Sigma \cup\{\tau\}$ there is a $\pi \in \Pi$ with $v^{+}(\pi)=v$. By (2) of Lemma 3.6, $\left|\Pi_{n}\right| \geq 2^{n-1}$ for every $n \geq 1$. This finishes the proof of case B1.

Case B2. There is a constant $L>0$ such that $\ell(u(\Pi)) \leq L$ and $\ell\left(v_{i, j}^{+}(\Pi)\right) \leq L$, $\ell\left(v_{i, j}^{-}(\Pi)\right) \leq L$ for every $1 \leq i \leq K, 1 \leq j \leq k-1$.

We prove the upper bound

$$
\left|\Pi_{n}\right| \leq F_{n, k-1} \cdot c_{1} n^{c_{2}} \text { for all } n \geq 1 \text {. }
$$

Every $\pi \in \Pi_{n}$ is uniquely determined by the word $u(\pi) \in[K]^{*}$ together with the $s_{k}(\pi) \leq$ $K$ restrictions $\pi \mid U_{i}$. For $s_{k}(\pi)<i \leq K$ we set $U_{i}=\emptyset$. Let $R(m)$ be the number of possible restrictions $\pi \mid U_{i}$ with $\left|U_{i}\right|=m$. If we prove that $R(m) \leq F_{m, k-1} \cdot c_{3} m^{c_{4}}$ for all $m \geq 1$ and constants $c_{3}, c_{4}>0$ (depending only on $K$ and $L$ ), we are done since (3) of Lemma 3.5 and (2) of Lemma 3.3 imply that

$$
\begin{aligned}
\left|\Pi_{n}\right| & \leq \sum_{u \in W_{K, L, n}} R\left(\left|U_{1}\right|\right) R\left(\left|U_{2}\right|\right) \ldots R\left(\left|U_{K}\right|\right) \quad\left(\text { note that }\left|U_{1}\right|+\cdots+\left|U_{K}\right|=n\right) \\
& \leq \sum_{u \in W_{K, L, n}} F_{n, k-1} \cdot c_{3}^{K} n^{c_{4} K} \\
& \leq F_{n, k-1} \cdot c_{3}^{K} n^{c_{4} K} \cdot(K+1)^{c_{5}} n^{c_{5}} \quad\left(\text { where } c_{5}=\left(\begin{array}{c}
K \\
2
\end{array}\right)(L-1)+1\right) \\
& \leq F_{n, k-1} \cdot c_{1} n^{c_{2}} .
\end{aligned}
$$

It remains to show that $R(m) \leq F_{m, k-1} \cdot c_{3} m^{c_{4}}$. Let $\sigma$ be a generic restriction $\pi \mid U_{i}$ with $\left|U_{i}\right|=m$ and $\pi$ ranging over $\Pi_{n}$. We have that $h^{+}(\sigma)<k$ or $h^{-}(\sigma)<k$; we may assume the former. For $1 \leq j \leq k-1$ we write $v_{j}^{+}(\sigma)$ for $v_{i, j}^{+}(\pi)$. By the hypothesis of case B2, $\ell\left(v_{j}^{+}(\sigma)\right) \leq L$ for every $1 \leq j \leq k-1$. Since $v^{+}(\sigma) \in\left(\operatorname{Ind}_{1}^{+} \cup \ldots \cup \operatorname{Ind}_{k-1}^{+}\right)^{*}$, we apply (3) of Lemma 3.3 and conclude that there is a partition into intervals

$$
v^{+}(\sigma)=J_{1} J_{2} \ldots J_{M} \text { with } M \leq 2\left(\begin{array}{c}
1 !+\cdots+(k-1) ! \\
2
\end{array}\right)(L-1)+2=N
$$

such that every $J_{i}$ uses at most one letter from any subalphabet $\operatorname{Ind}_{j}^{+}$. Let $Q(m)$ be the number of $\tau \in \Pi_{m}$ such that $h^{+}(\tau)<k$ and $v^{+}(\tau)$ uses at most one letter from every $\operatorname{Ind}_{j}^{+}$. Then, by (1) of Lemma 3.6,

$$
Q(m) \leq 1 ! \cdot 2 ! \cdot \ldots \cdot(k-1) ! \cdot F_{m, k-1}=c_{6} \cdot F_{m, k-1}
$$

because $v^{+}(\tau) \in \Sigma^{*}$ for a transversal $\Sigma$ of the $k-1$ sets $\operatorname{Ind}_{1}^{+}, \ldots, \operatorname{Ind}_{k-1}^{+}$, and there are at most $c_{6}$ such transversals.

So, by the bound on $Q(m)$, (3) of Lemma 3.5, and the bound $M \leq N$, the number of $\sigma$ 's satisfies

$$
R(m) \leq \sum_{m_{1}+\cdots+m_{N}=m} Q\left(m_{1}\right) Q\left(m_{2}\right) \ldots Q\left(m_{N}\right) \quad\left(\text { summing over } m_{i} \geq 0\right)
$$




$$
\begin{aligned}
& \leq \sum_{m_{1}+\cdots+m_{N}=m} F_{m, k-1} \cdot c_{6}^{N} \leq F_{m, k-1} \cdot c_{6}^{N}(m+1)^{N} \\
& \leq F_{m, k-1} \cdot c_{3} m^{c_{4}}
\end{aligned}
$$

This finishes the proof of case B2, of claim B, and of the whole theorem.

Every growth rate $n \mapsto F_{n, k}$ is attained by a closed class of permutations (take, for example, the permutations $\pi$ whose upward blocks are decreasing sequences of length at most $k$ ). This result was proved also by Egge in [16]. See [16] and Egge and Mansour [17] for many enumerative results on closed permutation classes involving the numbers $F_{n, k}$.

\section{Constant and polynomial growths}

We look in more detail at the slow growths and begin with the constant growth. Let $\pi$ be a permutation of $[n]$. For $r \in \mathbf{N}$, we say that $\pi$ has the $r$-intrusion property if there are subsets $X, Y \subset[n]$ and an element $x \in[n]$ such that $X<x<Y,|X|,|Y| \geq r$, and $\pi \mid(X \cup Y)$ is monotone but $\pi \mid(X \cup Y \cup\{x\})$ is not. We say that $\pi$ has the $r$-union property if there are subset $X, Y \subset[n]$ such that $X<Y,|X|,|Y| \geq r$, and both restrictions $\pi \mid X$ and $\pi \mid Y$ are monotone but $\pi \mid(X \cup Y)$ is not.

Lemma 4.1 Let $\Pi$ be any closed class of permutations.

(1) If for every $r \geq 1$ there is a $\pi \in \Pi$ such that $\pi$ or $\pi^{-1}$ has the $r$-intrusion property, then $\left|\Pi_{n}\right| \geq n$ for all $n \geq 1$.

(2) If for every $r \geq 1$ there is a $\pi \in \Pi$ with the $r$-union property, then $\left|\Pi_{n}\right| \geq n$ for all $n \geq 1$.

(3) Suppose $\pi \neq \tau$ are two permutations of $[n]$ and $I \subset[n]$ is such that all three sets $I, \pi(I)$, and $\tau(I)$ are intervals in $[n]$ and both restrictions $\pi \mid I$ and $\tau \mid I$ are monotone. Then for every subset $J \subset I$ such that $|I|-|J| \geq 2$ we have $\pi|([n] \backslash J) \neq \tau|([n] \backslash J)$.

Proof. (1) We may assume that for every $r \geq 1$ there is a $\pi \in \Pi_{2 r+1}$ such that $\pi \mid([2 r+$ $1] \backslash\{r+1\})$ is increasing but $\pi(r)>\pi(r+1)$; the other possible cases are very similar. Thus for every $n$ and $m, 1 \leq m \leq n-1$, there is a $\pi_{m} \in \Pi_{n}$ such that $\pi \mid[m]$ is increasing but $\pi(m)>\pi(m+1)$. The permutations $\pi_{m}$ are mutually distinct and together with $(1,2, \ldots, n) \in \Pi_{n}$ they show that $\left|\Pi_{n}\right| \geq n$.

(2) If $\pi,|\pi|=2 n$, is such that $\pi \mid[n]$ and $\pi \mid[n+1,2 n]$ are monotone but $\pi$ is not, then $\pi(n-1), \pi(n), \pi(n+1)$ or $\pi(n), \pi(n+1), \pi(n+2)$ is non-monotone. From this it easily follows, as in (1), that there are $n$ distinct permutations $\sigma,|\sigma|=n$, such that $\sigma \subset \pi$. Thus $\left|\Pi_{n}\right| \geq n$ for all $n \geq 1$.

(3) The restrictions of $\pi$ and $\tau$ on $[n] \backslash J$ must be different because at least 2 terms remained from the monotone sequences $\pi \mid I$ and $\tau \mid I$ and thus $\pi$ and $\tau$ can be completely reconstructed from the restrictions. 
Theorem 4.2 Let $\Pi$ be any closed class of permutations. Then exactly one of the following possibilities holds.

(1) $\left|\Pi_{n}\right|$ is constant for $n \geq n_{0}$.

(2) $\left|\Pi_{n}\right| \geq n$ for all $n \geq 1$.

Proof. We may assume that $\Pi$ is a closed permutation class such that, for some $r \geq 1$, for every $\pi \in \Pi$ neither $\pi$ nor $\pi^{-1}$ has the $r$-intrusion property and $\pi$ does not have the $r$-union property. If $r$ does not exist, we are done because (1) and (2) of Lemma 4.1 then imply that (2) holds. Now let $\pi \in \Pi_{n}$ be arbitrary and let $n \geq 9 r^{2}$. Consider the longest monotone subsequence of $\pi$, determined by $X \subset[n]$. Thus $\pi \mid X$ is monotone, $|X|$ is maximum, and, by Theorem 3.1, $|X| \geq 3 r$. We partition $X$ into the sets $X_{1}<Y<X_{2}$ where $\left|X_{1}\right|=\left|X_{2}\right|=r$. So $|Y| \geq r$. Since neither $\pi$ nor $\pi^{-1}$ has the $r$-intrusion property and $|X|$ is maximum, both $Y$ and $\pi(Y)$ must form an interval in $[n]$. Let $A=[\min Y-1] \backslash X_{1}$ and $B=[\max Y+1, n] \backslash X_{2}$. Using the assumption that $\pi$ does not have the $r$-union property and invoking Theorem 3.1, we see that $|A|,|B| \leq r^{2}$. We conclude that for every $\pi \in \Pi_{n}, n \geq 9 r^{2}$, there is a subset $Y \subset[n]$ such that $\pi \mid Y$ is monotone, both $Y$ and $\pi(Y)$ form an interval in $[n]$, and $n-|Y| \leq 2\left(r^{2}+r\right)=R$. If $R$ is enlarged to $R=9 r^{2}$, the conclusion holds for every $n \geq 1$.

For all $n \geq 1,\left|\Pi_{n}\right| \leq 2(R+1)^{2} R$ ! because we have two possibilities for $\pi \mid Y$, at most $R+1$ ways to place $Y$, the same number of ways to place $\pi(Y)$, and at most $R$ ! possibilities for $\pi \mid([n] \backslash Y)$. Let

$$
k=\limsup _{n \rightarrow \infty}\left|\Pi_{n}\right|
$$

and $n_{0} \in \mathbf{N}$ be such that $n_{0} \geq 2 R+2$ and $\left|\Pi_{n}\right| \leq k$ for $n \geq n_{0}$. We show that in fact, $\left|\Pi_{n}\right|=k$ for $n \geq n_{0}$. Let $n \geq n_{0}$ be arbitrary, $m \geq n$ be such that $\left|\Pi_{m}\right|=k$, and let $\Pi_{m}=\left\{\pi_{1}, \pi_{2}, \ldots, \pi_{k}\right\}$. These $k$ permutations satisfy the hypothesis of (3) of Lemma 4.1 with $I=[R+1, m-R]$. For $J=[n-R+1, m-R]$ we have $J \subset I,|I|-|J| \geq 2$, and $\sigma_{i}=\pi_{i} \mid([m] \backslash J) \in \Pi_{n}$ for $1 \leq i \leq k$. By (3) of Lemma 4.1, all $\sigma_{i}$ are distinct. Hence $\left|\Pi_{n}\right| \geq k$ and $\left|\Pi_{n}\right|=k$.

All possible constant growths are attained. The growth $n \mapsto 0$ is attained by $\Pi=\emptyset$ and $n \mapsto k, n \geq k$, is attained by $\{\pi \subset(1,2, \ldots, n, n+k, n+k-1, \ldots, n+1): n \in \mathbf{N}\}$. Similarly, $n \mapsto n$ is attained by $\{\pi \subset(1,2, \ldots, n, 2 n, 2 n-1, \ldots, n+1): n \in \mathbf{N}\}$. We were informed by M. Atkinson that the structure of closed classes with bounded growth, given in the previous proof, was also determined in Atkinson and Beals [6].

We proceed to the polynomial growth and partially characterize polynomially growing counting functions of closed permutation classes. For this, we need to look first at the partial order $\left(\mathbf{N}^{m}, \leq\right)$, where $m \in \mathbf{N}$ and $a=\left(a_{1}, \ldots, a_{m}\right) \leq\left(b_{1}, \ldots, b_{m}\right)=b$ means that $a_{i} \leq b_{i}$ for every $i=1, \ldots, m$. We say that a subset $S \subset \mathbf{N}^{m}$ is closed if $a \leq b \in S$ always implies $a \in S$. For $a \in \mathbf{N}^{m}$ we define $\|a\|=a_{1}+a_{2}+\cdots+a_{m}$. For a (closed) subset $S \subset \mathbf{N}^{m}$ and $n \in \mathbf{N}$ we set

$$
S_{n}=\{a \in S:\|a\|=n\} .
$$


The elements of $S_{n}$ can be represented by partitions of $[n]$ into $m$ nonempty intervals, and therefore the next lemma is a particular case of Theorem 3.1 in [19]. However, the direct proof is not too difficult; and for the sake of completeness, we give it here.

Lemma 4.3 For every closed set $S \subset \mathbf{N}^{m}$ there is a number $M \in \mathbf{N}$ and $(M+1)^{2}$ integers $a_{i, j}, 0 \leq i, j \leq M$, so that for every $n \geq n_{0}$ we have

$$
\left|S_{n}\right|=\sum_{i, j=0}^{M} a_{i, j}\left(\begin{array}{c}
n-i \\
j
\end{array}\right) .
$$

Proof. We say that $S \subset \mathbf{N}^{m}$ is canonical if there is an $m$-tuple $b \in(\mathbf{N} \cup\{\infty\})^{m}$ so that

$$
S=\left\{a \in \mathbf{N}^{m}: a_{i}<b_{i} \text { for every } i=1, \ldots, m\right\},
$$

where $a_{i}<\infty$ means that the $i$-th coordinate of $a$ is unrestricted. Canonical sets are closed and for a canonical $S$ determined by $b$ we have

$$
\left|S_{n}\right|=\left[x^{n}\right]\left(\frac{x}{1-x}\right)^{\#\left(b_{i}=\infty\right)} \prod_{b_{i}<\infty} \sum_{j=1}^{b_{i}-1} x^{j} \text { for } n \geq 1,
$$

where the empty sum equals 0 . If $b_{i}=\infty$ for no $i$, then $\left|S_{n}\right|=0$ for $n \geq n_{0}=\|b\|-$ $m+1$, and the formula is true with all $a_{i, j}$ zero. Otherwise, the formula follows by the binomial theorem. Thus the lemma holds in the case when $S$ is a canonical set, even with nonnegative $a_{i, j}$. It is clear that every intersection of canonical sets is again a canonical set. Therefore, by the inclusion-exclusion principle, the lemma holds more generally for every finite union of canonical sets (now we may get negative $a_{i, j}$ ).

Let $S \subset \mathbf{N}^{m}$ be any closed set. It suffices to show that $S$ is a finite union of canonical sets. $S$ is determined by the set $B$ of minimal elements in $\left(\mathbf{N}^{m} \backslash S, \leq\right)$. The set $B$ is an antichain. It is known, and not too difficult to prove, that every antichain in $\left(\mathbf{N}^{m}, \leq\right)$ is finite, see (for example) Nash-Williams [25, Lemma 1] for a more general result. So $B=\left\{b^{1}, b^{2}, \ldots, b^{r}\right\}$ and

$$
S=\left\{a \in \mathbf{N}^{m}: a \nsupseteq b^{i} \text { for every } i=1, \ldots, r\right\} .
$$

Thus

$$
a \in S \Longleftrightarrow \bigwedge_{i=1}^{r} \bigvee_{j=1}^{m}\left(a_{j}<b_{j}^{i}\right)
$$

The right hand side of the equivalence is equivalent to

$$
\bigvee_{j_{1}, \ldots, j_{r}} \bigwedge_{i=1}^{r}\left(a_{j_{i}}<b_{j_{i}}^{i}\right)
$$

where in the disjunction the $j_{i}$ 's range over all $r$-tuples from $[m]$. Since every conjuction $\bigwedge_{i=1}^{r}\left(a_{j_{i}}<b_{j_{i}}^{i}\right)$ defines a canonical set, $S$ is indeed a union of $m^{r}$ canonical sets and the lemma follows. 
A canonical form of a word $u$ is the form $u=u_{1}^{a_{1}} u_{2}^{a_{2}} \ldots u_{m}^{a_{m}}$. where $a_{i} \in \mathbf{N}, u_{i} \neq u_{i+1}$ for $i=1,2, \ldots, m-1$, and $u_{i}^{a_{i}}$ abbreviates $a_{i}$ repetitions of the letter $u_{i}$. Let $u(\pi)=$ $u_{1} u_{2} \ldots u_{n}$ be the word over $[m]$ determined by the 2-decomposition $U_{1}<U_{2}<\ldots<U_{m}$ of a permutation $\pi$ of length $n ; u_{i}=j \Leftrightarrow \pi^{-1}(i) \in U_{j}$ and every $\pi \mid U_{i}$ is monotone (see the proofs of Theorems 3.4 and 3.8). Recall that $\pi$ is uniquely determined by $u(\pi)$ and the $m$-tuple $s(\pi) \in\{+,-\}^{m}$ whose $i$-th component records whether $\pi \mid U_{i}$ is increasing or decreasing. Every $i<m$ appears in $u(\pi)$ at least twice and $i=m$ may appear only once. Let $u(\pi)=w_{1}^{a_{1}} w_{2}^{a_{2}} \ldots w_{r}^{a_{r}}$ be the canonical form of $u(\pi)$. The reduced form of $u(\pi)$ is the word

$$
u(\pi)^{*}=w=w_{1}^{b_{1}} w_{2}^{b_{2}} \ldots w_{r}^{b_{r}},
$$

where $b_{i}=1$ if $w_{i}=m$ or if $w_{j}=w_{i}$ for some $j \neq i$, else $b_{i}=2$. We define $E(w)=\{i \in$ $\left.[r]: b_{i}=2\right\}$ and $e(\pi) \in \mathbf{N}^{r}$ by $e_{i}=a_{i}$ if $i \notin E(w)$ and $e_{i}=a_{i}-1$ if $i \in E(w)$.

Let $\Pi$ be any closed permutation class. We split $\Pi$ into (non-closed) subclasses by the equivalence $\sim: \pi \sim \sigma$ iff $u(\pi)^{*}=u(\sigma)^{*}=w=w_{1}^{b_{1}} w_{2}^{b_{2}} \ldots w_{r}^{b_{r}}$ and $s(\pi)=s(\sigma)$. In one equivalence subclass $X$, the permutations are fully determined by the $r$-tuples

$$
e(X)=\{e(\pi): \pi \in X\} \subset \mathbf{N}^{r} .
$$

Note that $e(X)$ is closed (in the sense of the previous lemma) and that, for $\pi \in X$, $|\pi|=\|e(\pi)\|+|E(w)|$. By Lemma 4.3, there are $(M+1)^{2}$ integers $a_{i, j}$ such that

$$
\left|X_{n}\right|=\#\{\pi \in X:|\pi|=n\}=\sum_{i, j=0}^{M} a_{i, j}\left(\begin{array}{c}
n-i \\
j
\end{array}\right)
$$

for all $n \geq n_{0}$.

Theorem 4.4 If $\Pi$ is a closed class of permutations such that $\left|\Pi_{n}\right| \leq n^{c}$ for all $n \geq 1$ and a constant $c>0$, then there is a number $M \in \mathbf{N}$ and $(M+1)^{2}$ integers $a_{i, j}, 0 \leq i, j \leq M$, such that for all $n \geq n_{0}$ we have

$$
\left|\Pi_{n}\right|=\sum_{i, j=0}^{M} a_{i, j}\left(\begin{array}{c}
n-i \\
j
\end{array}\right) .
$$

Proof. The proof of Theorem 3.8 (case B2, $k=2$ ) shows that if $\left|\Pi_{n}\right| \leq n^{c}$ for all $n \geq 1$, then for all $\pi \in \Pi$ we have $m=s_{2}(\pi) \leq K$ and $\ell(u(\pi)) \leq L$ for some constants $K, L>0$. Thus by (1) of Lemma 3.3, the length of the reduced form of $u(\pi)$ is bounded by some $d>0$, and we have at most $(K+1)^{d}$ possible reduced forms $u(\pi)^{*}, \pi \in \Pi$. Hence the equivalence $\sim$ on $\Pi$ has at most $2^{K}(K+1)^{d}$ subclasses. We select $n_{0}$ large enough so that for every of the subclasses $X$ the above argument applies and for $n \geq n_{0}$ the number $\left|X_{n}\right|$ has the form $\sum_{i, j=0}^{M} a_{i, j}\left(\begin{array}{c}n-i \\ j\end{array}\right)$, with integral $a_{i, j}\left(M\right.$ and $a_{i, j}$ depend on $\left.X\right)$. Then for $n \geq n_{0}$ also the finite sum

$$
\left|\Pi_{n}\right|=\sum_{X}\left|X_{n}\right|
$$

has the form $\sum_{i, j=0}^{M} a_{i, j}\left(\begin{array}{c}n-i \\ j\end{array}\right)$. 
It is an interesting question to fully characterize those polynomials that can be realized (for $n \geq n_{0}$ ) as $n \mapsto\left|\Pi_{n}\right|$. For example, it follows from Theorem 4.2 that no polynomial

$\left(\begin{array}{c}n-i \\ 1\end{array}\right)=n-i, i \in \mathbf{N}$, can be realized as a counting function. Note that a part of Theorem 4.2, the fact that every bounded counting function must be eventually constant, is an immediate corollary of the last theorem.

\section{Concluding remarks}

The fact that the containment order of permutations admits an infinite antichain has been known for a long time. The earliest references are Laver [23, p. 9], Pratt [26], and Tarjan [32]. Kruskal [22, p. 304] mentions Laver's (counter)example four years earlier and Laver himself "[uses] a construction of Jenkyns and Nash-Williams" [18]. Thus the idea seems to go back to the late 1960s. The recent reference is Spielman and Bóna [29]. See Atkinson, Murphy and Ruškuc [7] for further results on permutation antichains.

Closed classes of permutations and their counting functions have been investigated before by Atkinson [5] who, together with West [34], gives the counting function $n \mapsto\left|\Pi_{n}\right|$ for every closed $\Pi$ of the form $\Pi=\operatorname{Forb}(\{\alpha, \beta\})$ where $|\alpha|=3,|\beta|=4$, and $\alpha \not \subset \beta$. Our approach is much inspired by the works of Scheinerman and Zito [28] and Balogh, Bollobás and Weinreich $[8,9,10]$ on the hereditary classes and monotone classes of graphs. (For graphs, hereditarity means that the class is 'closed' with respect to induced subgraphs, while monotonicity means that it is 'closed' with respect to all subgraphs.) As far as we know, graphs are the only combinatorial structures for which the counting functions of hereditary (closed) classes have been systematically investigated from a 'global' viewpoint. One global result (although cast in the 'local' $\operatorname{Forb}(X)$ language) on hereditary classes of set partitions is in Klazar [19, Theorem 3.1]. The counting functions of the hereditary classes of set partitions are further investigated in [21].

The question posed after Theorem 2.1, whether there are $2^{\aleph_{0}}$ closed permutation classes with counting functions mutually incomparable by the eventual dominance, has a positive answer for graph hereditary classes, see [9, Theorem 11]. Note also that if we restrict our attention to polynomially growing permutation classes, then by Theorem 4.4, the eventual dominance is a linear order.

\section{References}

[1] R. M. Adin and Y. Roichman, Shape avoiding permutations, J. Comb. Theory, Ser. A, 97 (2002), 162-176.

[2] M. H. Albert, M. D. Atkinson, C. C. Handley, D. A. Holton and W. Stromquist, On packing densities of permutations, Electr. J. Comb., 9 (2002), R5, 20 pages.

[3] N. Alon and E. Friedgut, On the number of permutations avoiding a given pattern, J. Comb. Theory, Ser. A, 89 (2000), 133-140. 
[4] R. Arratia, On the Stanley-Wilf conjecture for the number of permutations avoiding a given pattern, Electr. J. Comb., 6 (1999), N1, 4 pages.

[5] M. D. Atkinson, Restricted permutations, Discrete Math., 195 (1999), 27-38.

[6] M. D. Atkinson and R. Beals, Finiteness conditions on closed classes of permutations, unpublished manuscript, 1998.

[7] M. D. Atkinson, M. M. Murphy and N. Ruškuc, Partially well-ordered sets of permutations, Order, 19 (2002), 101-113.

[8] J. Balogh, B. Bollobás and D. Weinreich, The speed of hereditary properties of graphs, J. Comb. Theory, Ser. B, 79 (2000), 131-156.

[9] J. Balogh, B. Bollobás and D. Weinreich, The penultimate rate of growth for graph properties, Eur. J. Comb., 22 (2001), 277-289.

[10] J. Balogh, B. Bollobás and D. Weinreich, Measures on monotone properties of graphs, Discrete Appl. Math., 116 (2002), 17-36.

[11] M. Bóna, Exact and asymptotic enumeration of permutations with subsequence conditions, Ph.D. thesis, M.I.T, 1997.

[12] M. Bóna, Exact enumeration of 1342-avoiding permutations: a close link with labeled trees and planar maps, J. Comb. Theory, Ser. A, 80 (1997), 257-272.

[13] M. Bóna, Permutations avoiding certain patterns: the case of length 4 and some generalizations, Discrete Math., 175 (1997), 55-67.

[14] M. Bóna, The solution of a conjecture of Stanley and Wilf for all layered patterns, J. Comb. Theory, Ser. A, 85 (1999), 96-104.

[15] H. Davenport and A. Schinzel, A combinatorial problem connected with differential equations, Amer. J. Math., 87 (1965), 684-694.

[16] E. S. Egge, Restricted permutations related to Fibonacci numbers and $k$-generalized Fibonacci numbers, preprint arXiv:math.CO/0109219, 2001.

[17] E. S. Egge and T. Mansour, Restricted permutations, Fibonacci numbers, and $k$ generalized Fibonacci numbers, preprint arXiv:math.CO/0203226, 2002.

[18] T. A. Jenkyns and C. St. J. A. Nash-Williams, Counterexamples in the theory of well-quasi-ordered sets, Proof Techniques in Graph Theory, Proc. 2nd Ann Arbor Graph Theory Conference, 1968, Academic Press, New York 1969; pp. 87-91.

[19] M. Klazar, Counting pattern-free set partitions I: A generalization of Stirling numbers of the second kind, Eur. J. Comb., 21 (2000), 367-378. 
[20] M. Klazar, The Füredi-Hajnal conjecture implies the Stanley-Wilf conjecture, Proc. 12th international conference FPSAC'00, Moscow, 2000, Springer, Berlin 2000; pp. 250-255.

[21] M. Klazar, Counting pattern-free set partitions III: Growth rates of the hereditary classes, in preparation.

[22] J. B. Kruskal, The theory of well-quasi-ordering: A frequently discovered concept, J. Comb. Theory, Ser. A, 13 (1972), 297-305.

[23] R. Laver, Well-quasi-orderings and sets of finite sequences, Math. Proc. Camb. Philos. Soc., 79 (1976), 1-10.

[24] L. Lovász, Combinatorial Problems and Exercises, Akadémiai Kiadó, Budapest 1979.

[25] C. St. J. A. Nash-Williams, On well-quasi-ordering finite trees, Proc. Camb. Philos. Soc., 59 (1963), 833-835.

[26] V. R. Pratt, Computing permutations with double-ended queues, parallel stacks, and parallel queues, Proc. 5th Annual ACM Symposium on Theory of Computing, Assoc. Comput. Mach., New York 1973; pp. 268-277.

[27] A. Regev, Asymptotic values for degrees associated with strips of Young diagrams, Adv. Math., 41 (1981), 115-136.

[28] E. R. Scheinerman and J. Zito, On the size of hereditary classes of graphs, J. Comb. Theory, Ser. B, 61 (1994), 16-39.

[29] D. Spielman and M. Bóna, An infinite antichain of permutations, Electr. J. Comb., 7 (2000), N2, 4 pages.

[30] Z. Stankova-Frenkel and J. West, A new class of Wilf-equivalent permutations, $J$. Algebraic Combin., 15 (2002), 271-290.

[31] R. P. Stanley, Enumerative Combinatorics, Volume 1, Wadsworth \& Brooks/Cole, Monterey, Ca 1986.

[32] R. E. Tarjan, Sorting using networks of queues and stacks, J. Assoc. Comput. Mach., 19 (1972), 341-346.

[33] P. Valtr, private communication, January 2000.

[34] J. West, Generating trees and forbidden sequences, Dicrete Math., 157 (1996), 363374 .

[35] H. S. Wilf, The patterns of permutations, Discrete Math., 257 (2002), 575-583. 